\title{
High Resolution Structure of the Manganese Derivative of Insulin ${ }^{\dagger}$
}

\author{
Biserka Prugovečki, ${ }^{*}$ Ivana Pulić, Martina Toth, and Dubravka Matković-Čalogović \\ Division of General and Inorganic Chemistry, Department of Chemistry, Faculty of Science, \\ University of Zagreb, Horvatovac 102a, HR-10000 Zagreb, Croatia
}

RECEIVED JUNE 19, 2012; REVISED OCTOBER 2, 2012; ACCEPTED OCTOBER 3, 2012

\begin{abstract}
The structure of the human manganese derivative of insulin was determined using the single crystal X-ray diffraction method. Single crystals were grown by the hanging drop vapour diffusion crystallization method using zinc-free insulin and manganese(II) sulphate monohydrate in citrate buffer at $\mathrm{pH}=6.4$. It crystallizes in the trigonal system in the space group $R 3$ with the unit cell parameters: $a=b=$ $81.70 \AA$ and $c=33.73 \AA$. There are two manganese ions per insulin hexamer in the human manganeseinsulin derivative. The manganese ions lie on the three-fold crystallographic axis on opposite sides of the hexamer. Both manganese ions are octahedrally coordinated by three $\mathrm{N}^{\varepsilon 2}$ atoms from the imidazolyl side chains of three symmetry-related HisB10/HisD10 and three oxygen atoms of three symmetry-related water molecules. The investigated human manganese-insulin derivative adopts the $\mathrm{T}_{6}$ conformation. (doi: $10.5562 / \mathrm{cca} 2108)$
\end{abstract}

Keywords: insulin derivative, manganese, X-ray structure

\section{INTRODUCTION}

Insulin is a peptide hormone that regulates the level of glucose in the blood and is a therapeutic protein that is used medically for the treatment of diabetes mellitus. Insulin is structured of two polypeptide chains with chain A consisting of 21 and chain $B$ of 30 amino acids. Chains A and B are linked by two disulphide bonds, while the third intrachain disulphide bond links residues A6 and A11. Metal ions have important implications on insulin storage, release and conformation. Insulin storage vesicles in many species contain high concentrations of $\mathrm{Zn}^{2+}$ and $\mathrm{Ca}^{2+}$ ions and insulin is accumulated in the pancreas as a $\mathrm{Zn}^{2+}$ containing hexamer. Hexamer formation is related to insulin storage and to some of processes in its biosynthesis. ${ }^{1-3}$ There are three forms of insulin hexamers named $T_{6}, T_{3} R_{3}{ }^{f}$ and $R_{6}$. These notations refer to the folding of the N-terminal part of the $\mathrm{B}$ chains in insulin hexamers. ${ }^{4}$ The $\mathrm{T}_{6}$ conformation is obtained at low chloride concentrations and in absence of phenolic derivatives. ${ }^{5-7}$ The $\mathrm{T}_{6}$ insulin hexamer has two high-affinity metal ion binding sites which are located on the 3-fold axis and each metal ion is octahedrally coordinated by three His B10 residues (one from each dimer) and three water molecules. It has been shown that chloride ions induce the $\mathrm{T}$ to $\mathrm{R}$ transition at residues $\mathrm{B} 4-\mathrm{B} 8$ in three molecules out of six in the insu- lin hexamer forming the $\mathrm{T}_{3} \mathrm{R}_{3}{ }^{\mathrm{f}}$ hexamer. ${ }^{8,9}$ It was found that $T_{3} R_{3}{ }^{f}$ type of hexamer can contain a different number of $\mathrm{Zn}^{2+}$ ions per hexamer with different coordination. When phenol or phenolic derivatives are included in the crystallization solution, all six B chain $\mathrm{N}$ termini adopt a $\alpha$-helical conformation, termed the $\mathrm{R}_{6}$ hexamer form. ${ }^{10-13}$ Many structural studies on chemically and genetically modified insulins have been done. It has been known for many years that different metal ions can substitute zinc ions in the $\mathrm{T}_{6}$ hexamer. ${ }^{14}$ The zinc binding cavities in the insulin hexamer can accommodate different metal ions which prefer imidazole and water molecule ligands. ${ }^{15-19}$ As a part of our ongoing research on the crystallization and structural studies on human insulin derivatives ${ }^{17,18}$ in the present study the zinc ions in human insulin were substituted with manganese.

Manganese is a trace mineral that takes part in wide range of metabolic function. The human body contains approximately ten miligrams of manganese, and it is a cofactor for a number of enzymes like arginase, pyruvate carboxylase, mitochondrial superoxide dismutase and several peptidases and glycosyltransferases. ${ }^{20-22}$ Prothrombin and vitamin $\mathrm{K}$ are formed with the help of manganese and it is also important for the body's utilization of vitamin E.

The crystal structure of $\mathrm{Mn}^{2+}$ arg-insulin hexamer (a modified form of insulin in which an arginine residue

\footnotetext{
$\dagger$ This article belongs to the Special Issue devoted to the $85^{\text {th }}$ anniversary of Croatica Chemica Acta.

* Author to whom correspondence should be addressed. (E-mail: biserka@chem.pmf.hr)
} 
is attached to the N-terminal of the A-chain of human insulin) was recently reported as a $\mathrm{T}_{3} \mathrm{R}_{3}{ }^{\mathrm{f}}$ hexamer. ${ }^{15} \mathrm{We}$ report here the crystal structure of hexameric $\mathrm{T}_{6}$ human manganese-insulin derivative.

\section{EXPERIMENTAL}

\section{Crystallization}

Crystals of the human manganese-insulin derivative were grown by the hanging drop vapour diffusion method. Chemicals employed in the crystallization study were reagent grade or better and were used without further purification. Zinc-free biosynthetic human insulin was used. Optimum crystallization conditions are as follows: the protein solution consisted of $7.5 \mathrm{mg} \mathrm{mL}^{-1}$ of zinc-free insulin in $0.02 \mathrm{~mol} \mathrm{~L}^{-1} \mathrm{HCl}$; the reservoir solution was at $\mathrm{pH}=6.4$ containing sodium citrate $\left(1 \mathrm{mmol} \mathrm{L}^{-1}\right)$, acetone $(\varphi=10 \%), \mathrm{MnSO}_{4} \cdot \mathrm{H}_{2} \mathrm{O}$ $\left(9 \mathrm{mmol} \mathrm{L}^{-1}\right)$ and redistilled water. Each drop consisted of $1 \mu \mathrm{L}$ of protein solution and $1 \mu \mathrm{L}$ of reservoir solution. Colourless, sharp-edged rhombohedrons up to $0.4 \mathrm{~mm}$ in length grew in 4 days at $T=291 \mathrm{~K}$.

\section{Diffraction Data Collection}

Intensity data for the human manganese-insulin derivative were collected on a single crystal cryoprotected by immersing it in the solution consisting of a volume fraction of $\varphi=70 \%$ reservoir solution and $\varphi=30 \%$ ethylene-glycol. The low temperature diffraction data to $1.08 \AA$ resolution were collected with oscillation angles of $1.0^{\circ}$ at the ELETTRA Synchrotron Light Laboratory, beam-line XRD-1 (wavelength, $\lambda=1.00 \AA$ ) using a CCD detector from MAR Research and an Oxford Cryosystems cryocooler. The data were processed with MOSFLM $^{23}$ and then scaled and merged by use of the CCP4 suite. ${ }^{24}$ Data statistics are given in Table 1.

\section{X-ray Analysis and Structure Refinement}

The $\mathrm{T}_{6}$ human nickel insulin derivative ${ }^{17}$ from which all water molecules, nickel ions and alternate side chains were omitted was taken as a starting model for refinement. Initially only $4 \AA$ resolution data were included in the rigid body refinement. The largest peaks from the initial $F_{\mathrm{o}}-F_{\mathrm{c}}$ map corresponded to a pair of manganese atoms located on the three-fold axis. The structure was then refined at a higher resolution using maximumlikelihood minimization implemented in REFMAC ${ }^{25}$ with $5 \%$ of the total data being excluded for the purpose of the $R_{\text {free }}$ refinement. Program COOT $^{26}$ was used to fit the model into the electron density map $\left(2 F_{\mathrm{o}}-F_{\mathrm{c}}\right.$ and $F_{\mathrm{o}}-F_{\mathrm{c}}$ maps). Water molecules were added by the $\mathrm{ARP} / \mathrm{wARP}$ program ${ }^{27}$ and were checked in accord with the criteria of electron density and acceptable hydrogen
Table 1. Data measurement and refinement statistics for the $\mathrm{T}_{6}$ human manganese-insulin derivative

\begin{tabular}{|c|c|}
\hline Space group & $R 3$ \\
\hline \multicolumn{2}{|l|}{ Unit cell parameters } \\
\hline$a=b / \AA$ & 81.70 \\
\hline$c / \AA$ & 33.73 \\
\hline$\alpha=\beta / \circ$ & 90 \\
\hline$\gamma /{ }^{\circ}$ & 120 \\
\hline Temperature / K & 100 \\
\hline$\left\langle B_{\text {iso }}\right\rangle / \AA^{2}$ & 8.583 \\
\hline No. of frames & 120 \\
\hline Resolution / $\AA$ & $10.3-1.08$ \\
\hline Completeness / \% & 96.7 \\
\hline No. of unique reflections & 43875 \\
\hline No. of free reflections & 1770 \\
\hline Overall $R_{\text {merge }}$ & 0.089 \\
\hline $\operatorname{Mean}(I / \sigma I)$ & 11.0 \\
\hline \multicolumn{2}{|l|}{ Refinement statistic } \\
\hline$R$ & 0.159 \\
\hline$R_{\text {free }}$ & 0.194 \\
\hline Cross-validated $\sigma_{\mathrm{A}}$ estimated error $/ \AA$ & 0.129 \\
\hline \multicolumn{2}{|l|}{ R.m.s. deviations from ideal } \\
\hline Bond lengths / $\AA$ & 0.027 \\
\hline Bond angles $/{ }^{\circ}$ & 2.4 \\
\hline Dihedral angles $/{ }^{\circ}$ & 7.0 \\
\hline \multicolumn{2}{|l|}{ Highest resolution shell } \\
\hline Resolution range / $\AA$ & $1.108-1.080$ \\
\hline No. of reflections & 5824 \\
\hline Completeness / \% & 87.3 \\
\hline$R$ & 0.233 \\
\hline$R_{\text {free }}$ & 0.299 \\
\hline $\operatorname{Mean}(I / \sigma I)$ & 6.3 \\
\hline
\end{tabular}

bonds to other atoms. The final refinement was carried out using anisotropic displacement parameters. Side chains SerA9, ValB12, ThrB27, SerD9, ValD12 were refined in two alternative conformations with occupancies of $50 \%$. Low density was observed for the terminal atoms of the side chain of PheD1, ValD2 and LysD29. The refinement converged at $R=0.159$ and $R_{\text {free }}=$ 0.194 . The final model consists of 844 protein atoms, 189 water molecules, two manganese, and one sodium ion. The model has good stereochemistry, the r.m.s. deviations for bond lengths and bond angles are $0.027 \AA$ and $2.4^{\circ}$, respectively. In the Ramachandran plot $97.53 \%$ of the residues are in the preferred regions and $2.47 \%$ in allowed regions. The crystallographic data and structure 

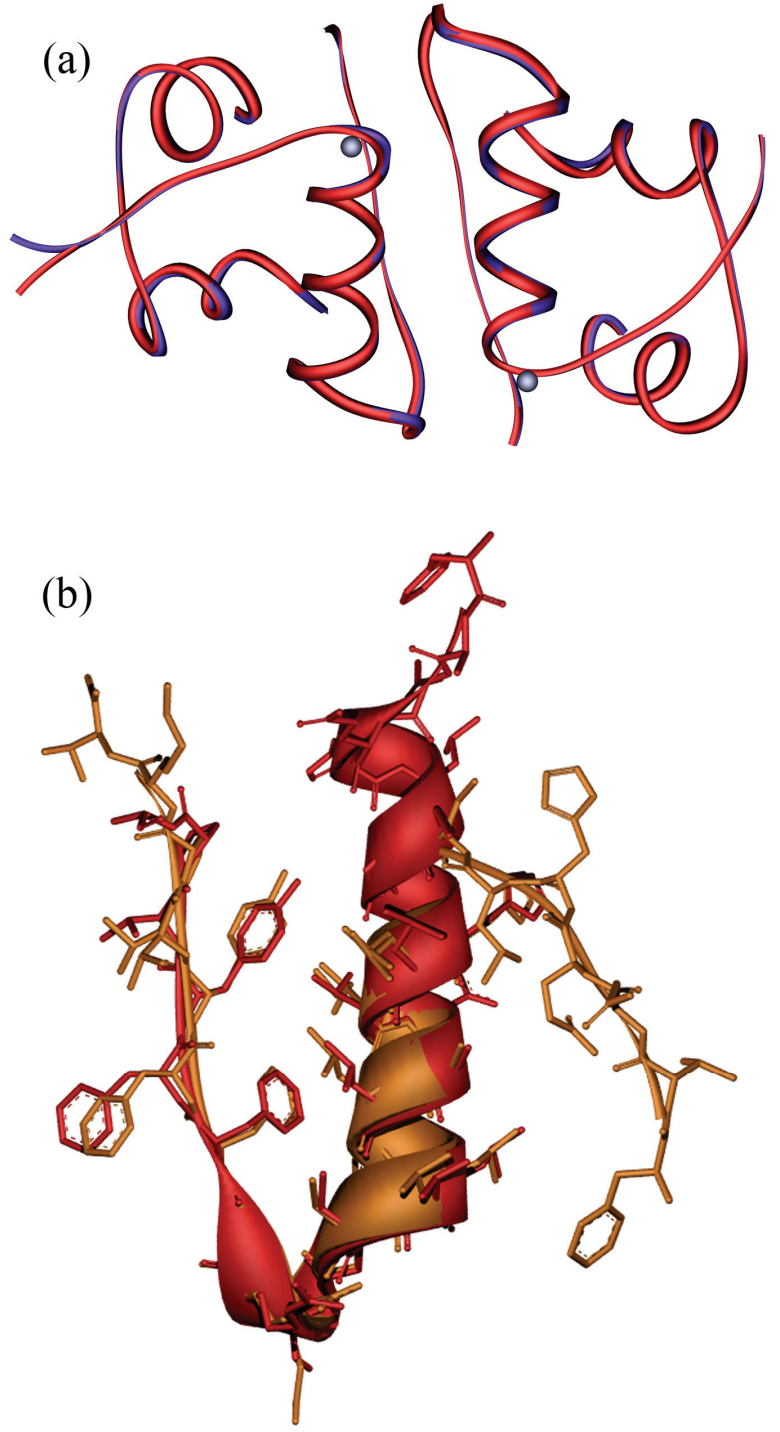

Figure 1. Cartoon representation of the superposed $\mathrm{T}_{2} \mathrm{Mn}^{2+}$ insulin derivative (red) and $\mathrm{T}_{2} \mathrm{Zn}^{2+}$ insulin derivative (blue). Manganese and zinc ions are shown as spheres (a). The cartoon representation of the superposed $B$ chain of the $T_{3} R_{3}{ }^{f}$ $\mathrm{Mn}^{2+}$ arg-insulin (red) with the same chain in the the $\mathrm{T}_{6} \mathrm{Mn}^{2+}$ insulin (orange) (b). refinement statistics are given in Table 1 . The geometry of the model was monitored with PROCHECK. ${ }^{28}$ The structure drawings were done by the PyMOL $^{29}$ program and Discovery Studio 3.1 Visualizer. ${ }^{30}$

\section{RESULTS AND DISCUSSION}

\section{Dimer and Hexamer Conformation}

Unlike the crystal structure of the $\mathrm{Mn}^{2+}$ arg-insulin ${ }^{15}$ hexamer which was reported as $\mathrm{T}_{3} \mathrm{R}_{3}{ }^{\mathrm{f}}$, the investigated human manganese-insulin derivative adopts a $\mathrm{T}_{6}$ conformation like in the native $\mathrm{Zn}^{2+}$ insulin derivative. ${ }^{6}$ The superposition of the $\mathrm{T}_{2} \mathrm{Mn}^{2+}$ insulin derivative and $\mathrm{T}_{2}$ native $\mathrm{Zn}^{2+}$ insulin derivative is shown in Figure 1a, while the superposition of the $B$ chain of the $T_{3} R_{3}{ }^{f} \mathrm{Mn}^{2+}$ arg-insulin with the $\mathrm{B}$ chain of $\mathrm{T}_{6} \mathrm{Mn}^{2+}$ insulin derivative is shown in Figure 1b.

In the crystal structure the asymmetric unit consists of one $T_{2}$ dimer resulting in three $T_{6}$ insulin hexamers in the unit cell. The dimer is asymmetrical because the B25Phe side chain turns away from the two fold axis and contacts D25Phe across the two-fold axis. The same asymmetry at B25 is present in the native $\mathrm{T}_{6} \mathrm{Zn}^{2+}$ insulin ${ }^{6}$. The root mean square displacement between the $T_{2}$ dimer of investigated human manganeseinsulin derivative and the $\mathrm{TR}^{\mathrm{f}} \mathrm{Mn}^{2+}$ arg-insulin derivative calculated using the CCP4 program LSQKAB ${ }^{31}$ is given in Table 2, indicating the major structural difference in the $\mathrm{B}$ chain of the dimer molecules. Chains $A, B$, and $C$ in the native $T_{2}$ zinc $\operatorname{dimer}^{6}$ and the $T_{2}$ dimer of investigated human manganese-insulin derivative are similar within the limits of experimental error. The greatest main chain difference between those structures was found in the D chain (Table 2).

\section{Coordination of Manganese Ions}

It is well known that the $\mathrm{T}_{6}$ insulin hexamer with $\mathrm{Zn}$ ions has two high-affinity metal ion binding sites which are located on the 3-fold axis and each metal ion is octahedrally coordinated by three $\mathrm{N}^{\varepsilon 2}$ atoms from the

Table 2. Root mean square differences (expressed in $\AA$ ) between the $T_{2}$ dimer of the investigated human manganese-insulin derivative, the $\mathrm{TR}^{\mathrm{f}} \mathrm{Mn}^{2+}$ arg-insulin derivative and the $\mathrm{T}_{2}$ zinc insulin

\begin{tabular}{ccccccccc}
\hline & \multicolumn{3}{c}{$\mathrm{TR}^{\mathrm{f}} \mathrm{Mn}^{2+}$ arg-insulin } & & \multicolumn{3}{c}{$\mathrm{T}_{2}$ zinc insulin } \\
\cline { 2 - 4 } Residue range & All atoms & $\mathrm{C}^{\alpha}$ atoms & Main chain & & All atoms & $\mathrm{C}^{\alpha}$ atoms & Main chain \\
\hline A chain & 1.65 & 1.14 & 1.19 & & 0.76 & 0.07 & 0.24 \\
B chain & 6.67 & 5.84 & 5.68 & & 0.86 & 0.10 & 0.12 \\
C chain & 1.29 & 0.42 & 0.46 & & 0.46 & 0.10 & 0.20 \\
D chain & 3.20 & 2.70 & 2.50 & & 1.40 & 1.13 & 1.19 \\
\hline
\end{tabular}


Table 3. Bond lengths (expressed in $\AA$ ) between the octahedrally coordinated metal ions $\left(\mathrm{Mn}^{2+}\right.$ or $\left.\mathrm{Zn}^{2+}\right)$ and $\mathrm{N}^{\varepsilon 2}$ atoms from the imidazolyl side chains of three HisB10 / HisD10 and oxygen atoms of water molecules in $\mathrm{T}_{2}$ dimer of the investigated human manganese-insulin derivative and the $T_{2}$ zinc insulin

\begin{tabular}{llrll}
\hline & $\mathrm{T}_{2} \mathrm{Mn}^{2+}$ insulin & \multicolumn{2}{c}{$\mathrm{T}_{2} \mathrm{Zn}^{2+}$ insulin } \\
\hline $\mathrm{Mn}-\mathrm{N}^{\varepsilon 2}$ & 2.09 & 2.14 & & \\
$\mathrm{Mn}-\mathrm{O}$ & 2.19 & 2.21 & & \\
$\mathrm{Zn}-\mathrm{N}^{\varepsilon 2}$ & & & 2.09 & 2.10 \\
$\mathrm{Zn}-\mathrm{O}$ & & & 2.20 & 2.23 \\
\hline
\end{tabular}

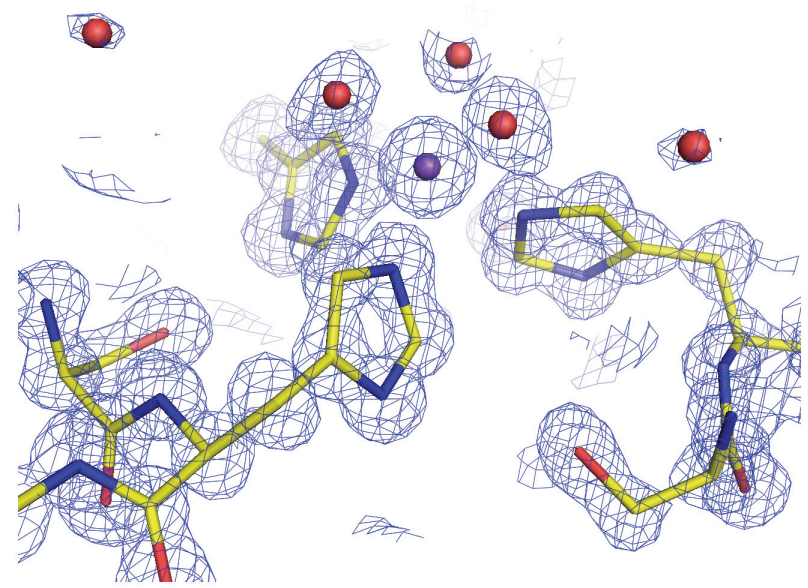

Figure 2. Coordination around the manganese ion showing the electron density: manganese atom (violet) is octahedrally coordinated by three $\mathrm{N}^{\varepsilon 2}$ atoms from the imidazolyl side chains of three symmetry-related HisB10 and three oxygen atoms of three symmetry-related water molecules (red). The $2 F_{o}-F_{c}$ map is contoured at $1.4 \sigma$.

imidazolyl side chains of three symmetry-related HisB10/HisD10 and three oxygen atoms of three symmetry-related water molecules. ${ }^{5-7,16-18}$ The same coordination is found in the present $\mathrm{Mn}^{2+}$ insulin structure (Figure 2). So far, only in $\mathrm{T}_{6} 4 \mathrm{Ni}^{2+}$ human arg-insulin crystal structure a dual octahedral and tetrahedral coordination of nickel ion is found at one site. ${ }^{15}$ In the $\mathrm{Mn}^{2+}$ arg-insulin structure a tetrahedral coordination of $\mathrm{Mn}$ ion is observed (three $\mathrm{N}^{\varepsilon 2}$ atoms from the imidazolyl side chains of three symmetry-related HisB10/HisD10 and a chloride ion). ${ }^{15}$ Bond lengths between the octahedrally coordinated metal ions $\left(\mathrm{Mn}^{2+}\right.$ or $\left.\mathrm{Zn}^{2+}\right)$ and $\mathrm{N}^{\varepsilon 2}$ atoms from the imidazolyl side chains of three HisB10 / HisD10 and oxygen atoms of water molecules in $T_{2}$ dimer of the investigated human manganese-insulin derivative and the $T_{2}$ zinc insulin are very similar (Table 3). The B10 to D10 manganese distance found in the $\mathrm{T}_{6}$ manganese derivative is $16.44 \AA$ while in the $\mathrm{Mn}^{2+}$ arg-insulin structure it was found to be $15.92 \AA$ and in the native $T_{6}$ human insulin the distance between two zinc ions is $16.42 \AA$.
An analysis of the Protein Data Bank ${ }^{32}$ with the crystal structures containing an octahedrally coordinated manganese ion with histidine as a ligand was carried out using the MESPEUS, ${ }^{33}$ a database of the geometry of metal sites in proteins. Only structures with resolution $\leq 2.0 \AA$ were investigated. There were 463 hits having octahedrally coordinated manganese ion with histidine as a ligand of which 29 structures had three histidines as ligands. In 9 structures three histidines and one or two water molecules were found as ligands to manganese.

The Cambridge Structural DataBase ${ }^{34}$ search using the ConQeust Version 1.14, gave 15 structures of small molecules containing octahedrally coordinated manganese ion with three water molecules and three $\mathrm{N}$-donor atoms. The $\mathrm{Mn}-\mathrm{N}$ bond lengths are in the range of 2.117-2.431 $\AA$ and the $\mathrm{Mn}-\mathrm{O}$ bond lengths in those structures are in the range of $2.128-2.349 \AA$.

\section{CONCLUSION}

The results show that the investigated manganeseinsulin derivative adopts the $\mathrm{T}_{6}$ conformation with two manganese ions located on the 3 -fold axis separated by a distance of $16.44 \AA$. Both manganese ions are octahedrally coordinated by three $\mathrm{N}^{\varepsilon 2}$ atoms from the imidazolyl side chains of three symmetry-related HisB10/HisD10 and three oxygen atoms of three symmetry-related water molecules. $\mathrm{T}_{6}$ form of manganeseinsulin derivative was compared with the $\mathrm{T}_{3} \mathrm{R}_{3}{ }^{\mathrm{f}}$ form of the $\mathrm{Mn}^{2+}$ arg-insulin structure and difference in hexamer conformation as well as metal coordination was found.

Supplementary Materials. - The coordinates and structure factor data for the reported manganese-insulin derivative have been submitted to the Protein Data Bank; PDB code 4FKA.

Acknowledgements. Financial support for this research was provided by the Ministry of Science, Education and Sports of the Republic of Croatia (Grant No. 119-1193079-1084). We thank professor Guy Dodson for his kind donation of zinc-free insulin.

\section{REFERENCES}

1. S. L. Howell, W. Montague, and M. Tyhurst, J. Cell Sci. 19 (1975) 395-409.

2. J. Brange, (1994) Studies on insulin stability, Stability of Insulin, Kluwer, Academic Publishers, Dordrecht, The Netherlands, pp.17-37.

3. G. G. Dodson and D. Steiner, Curr. Opin. Struct. Biol. 8 (1998) 189-194.

4. N. C. Kaarsholm, H. C. Ko, and M. F. Dunn, Biochemistry 28 (1989) 4427-4435.

5. M. J. Adams, T. L. Blundell, E. J. Dodson, G. G. Dodson, M. Vijayan, F. H. Allen, J. E. Davies, J. J. Galloy, O. Johnson, O. Dennard, E. N. Baker, M. M. Harding, D. C. Hodgkin, R. Rimmer, and S. Sheet, Nature 224 (1969) 491-495. 
6. D. G. Smith, W. A. Pangborn, and R. H. Blessing, Acta Crystallogr., Sect. D 59 (2003) 474-482.

7. D. G. Smith, W. A. Pangborn, and R. H. Blessing, Acta Crystallogr., Sect. D 61 (2005) 1476-1482.

8. G. Bentley, E. Dodson, G. Dodson, D. Hodkin, and D. Mercola, Nature 261 (1976) 166-168.

9. E. Ciszak and G. D. Smith, Biochemistry 33 (1994) 1512-1517.

10. J. L. Whittingham, S. Chaudhuri, E. J. Dodson, P. C. E. Moody, and G. G. Dodson, Biochemistry 34 (1995) 15553-15563.

11. G. D. Smith, E. Ciszak, and W. Pangborn, Protein Sci. 5 (1996), 1502-1511.

12. G. D. Smith, E. Ciszak, L. A. Magrum, W. A. Pangborn and R. H. Blessing, Acta Crystallogr., Sect. D 56 (2000) 1541-1548.

13. M. Norman and G. Schluckebier, BMC Structural Biology (2007).

14. C. P. Hill, Z. Dauter, E. J. Dodson, G. G. Dodson, and M. F. Dunn, Biochemistry 30 (1991) 917-924.

15. R. Sreekanth, V. Pattabhi, and S. S. Rajan, Int. J. Biol. Macromol. 44 (2009) 29-36.

16. N. R. S. Krishna, V. Pattabhi, and S. S. Rajan, Protein Pept. Lett. 18 (2011) 457-466.

17. B. Prugovečki, E. J. Dodson, G. G. Dodson, and D. MatkovićČaligović, Croatica Chemica Acta 82 (2009) 433-438.

18. B. Prugovečki, PhD Thesis, University of Zagreb, 2005.

19. J. Nicholson, L. Perkins, and F. Körber, Recent Res. Dev. Mol. Biol. 3 (2006) 1-16.

20. L. Di Costanzo, G. Sabio, A. Mora, P. C. Rodriguez, A. C. Ochoa, F. Centeno, D. W. Christianson, Proc. Natl. Acad. Sci. U.S.A. 102 (37) (2005) 13058-13063.

21. S. Jitrapakdee, M. St Maurice, I. Rayment, W. W. Cleland, J. C.
Wallace, and P. V. Attwood, Biochem. J. 413 (2008) 369-387.

22. G. E. Borgstahl, H. E. Parge, M. J. Hickey, M. J. Johnson, M. Boissinot, R. A. Hallewell, J. R. Lepock, D. E. Cabelli, J. A. Tainer, Biochemistry 35 (1996). 4287-4297.

23. A. G. W. Leslie, P. Brick, and A. J. Wonacott, CCP4 Newslett. 18 (1986) 33-39.

24. CCP4 Collaborative Computational Project, Number 4. "The CCP4 Suite: Programs for Protein Crystallography" Acta Crystallogr., Sect. D 50 (1994) 760-763.

25. G. N. Murshudov, A. A. Vagin, and E. J. Dodson, Acta Crystallogr., Sect. D 53 (1997) 240-255.

26. V. S. Lamzir, A. Perrakis, and K. S. Wilson, International Tables for Crystallography, Crystallography of Biological Macromolecules, Kluwer Academic Publisher, Dordrecht, 1999, pp.720-722.

27. G. G. Langer, S. X. Cohen, A. Perrakis, and V. S. Lamzin, Nature Protocols 3 (2008) 1171-1179.

28. R. A. Laskowski, M. W. MacArthur, D. S. Moss and J. M. Thornton, J. Appl. Crystallogr. 26 (1993) 283-291.

29. W. L. DeLano, The PyMOL Molecular Graphics System (2002) http://www.pymol.org

30. Accelrys Software Inc., Discovery Studio Modeling Environment, Release 3.1, San Diego: Accelrys Software Inc., 2012

31. W. Kabsch, Acta Crystallogr., Sect A 32 (1976) 922-923.

32. H. M. Berman, J. Westbrook, Z. Feng, G. Gilliland, T. N. Bhat, H. Weissig, I. N. Shindyalov and P. E. Burne, Nucleic Acid Res. 28 (2000) 235-242. The protein DataBank, http://www.rcsb.org

33. K. Hsin, Y. Sheng, M. M. Harding, P. Taylor, and M. D. Walkinshaw, J. Appl. Crystallogr. 41 (2008) 963-968.

34. F. H. Allen, Acta Crystallogr., Sect. B 58 (2002) 380-388. 